\title{
Severe Sprue-Like Enteropathy and Colitis due to OImesartan: Lessons Learned From a Rare Entity
}

\author{
Daniel R. Bashari
}

\begin{abstract}
Olmesartan is an angiotensin II receptor blocker (ARB) drug approved in 2002 for the treatment of hypertension. Since 2012, there have been reports of a rare adverse effect suspected to be related to its use. The author presents a case of a 63 -year-old female with refractory gastrointestinal (GI) symptoms including diarrhea with associated weight loss and severe electrolyte abnormalities necessitating hospitalization. An extensive inpatient evaluation ensued and was initially unremarkable. Esophagogastroduodenoscopy (EGD) discovered an endoscopically normal duodenum that was biopsied and notably revealed villous atrophy and intraepithelial lymphocytosis. Colonoscopy was normal appearing though biopsy findings were significant for nonspecific colitis. The endoscopy findings in the setting of the clinical presentation confirmed the diagnosis of olmesartanassociated enteropathy (OAE). Clinical improvement was noted after cessation of olmesartan and histological resolution was confirmed with repeat EGD post-discharge.
\end{abstract}

Keywords: Olmesartan medoxomil; Angiotensin II receptor blocker; Drug-induced enteropathy; Adverse drug reaction; Enteropathy; Drug-induced diarrhea; Villous atrophy; Enterocolitis

\section{Introduction}

Sprue-like enteropathy is an entity which is characterized by gastrointestinal (GI) symptoms including diarrhea in combination with histological findings of abnormal villous architecture of the small intestine $[1,2]$. Causes of villous atrophy and enteropathy have been largely attributed to celiac disease, autoimmune enteropathy, and drug-induced enteropathy $[1$, 2]. Drug-induced enteropathy has classically been associated with immunosuppressants and non-steroidal anti-inflammatory drugs (NSAIDs), though since 2012 there have been reports of a sprue-like enteropathy associated with the widely

Manuscript submitted June 1, 2020, accepted July 7, 2020

Published online August 14, 2020

Division of Hospital Medicine, Department of Medicine, Greater Baltimore Medical Center, 6701 N. Charles St., Baltimore, MD 21204, USA.

Email: danielbasharimd@gmail.com

doi: https://doi.org/10.14740/gr1301 prescribed antihypertensive drug olmesartan [2]. These cases have proven to be particularly challenging to diagnose in a timely manner as patients may experience this rare adverse effect months to years after initiation of the drug [1]. Herein, is a case of a 63-year-old female presenting to an academic community hospital with a 3-week history of diarrhea and unintentional weight loss in which olmesartan-associated enteropathy $(\mathrm{OAE})$ and colitis were ultimately diagnosed.

\section{Case Report}

A 63-year-old female presented to the hospital in March 2016, with a 3-week history of diarrhea. The patient had reported 10 - 20 daily episodes of diarrhea, described as watery, occurring at any time of the day, and not affected by food intake. The diarrhea was not associated with any blood loss or mucus production. Associated symptoms included nausea and unintentional weight loss of 10 pounds over the preceding week. She reported early satiety with minimal food or drink intake followed by a sensation of bloating. The patient denied any abdominal pain, fever, or chills. She denied consuming any new, exotic, or undercooked food in the recent past. Prior to her presentation, she had seen her primary care physician and had stool studies collected. Stool for clostridium difficile polymerase chain reaction (PCR) and bacterial culture both returned negative. She was empirically prescribed ciprofloxacin and loperamide without improvement in her symptoms. She was then referred to her gastroenterologist who then subsequently directed her to the hospital for an inpatient workup.

Past medical history included hypertension, anxiety, and depression. Surgical history included an appendectomy and a salpingo-oophorectomy for endometriosis. Her home medications, none of which were recently initiated, included olmesar$\tan /$ hydrochlorothiazide $40 / 12.5 \mathrm{mg}$ daily and fluoxetine 10 $\mathrm{mg}$ daily. There was no antibiotic or NSAID use that preceded her symptoms. She had not been in contact with anyone else who had been ill. She reported living by herself, having no pets, and living in a home that used well water. She denied any recent travel. Drug allergies included intolerance to codeine. Family history was notable for a history of colon cancer in her maternal grandmother. She denied any smoking or alcohol use.

Physical examination was unremarkable including a normal abdominal exam without tenderness or distention. Vital signs on arrival were as follows: blood pressure 121/69 mm $\mathrm{Hg}$, pulse $52 \mathrm{bpm}$, respiratory rate $20 / \mathrm{min}$, temperature 36.9 ${ }^{\circ} \mathrm{C}$, and oxygen saturation $99 \%$ on ambient air. Initial laborato- 
ry studies on admission were notable for the following: white blood cells $8.30 \times 10^{3} / \mu \mathrm{L}$, hemoglobin $15.8 \mathrm{~g} / \mathrm{dL}$, sodium 140 $\mathrm{mEq} / \mathrm{L}$, potassium $2.5 \mathrm{mEq} / \mathrm{L}$, and bicarbonate $18.3 \mathrm{mEq} / \mathrm{L}$ with a corresponding elevated anion gap of $17.7 \mathrm{mEq} / \mathrm{L}$. Liver function tests were unremarkable besides a mildly elevated of alanine transaminase (ALT) of $51 \mathrm{IU} / \mathrm{L}$. Albumin and total protein were within normal limits at 4.7 and $8.2 \mathrm{~g} / \mathrm{dL}$, respectively. An abdominal X-ray on the day of admission showed a nonspecific bowel gas pattern in both the small and large bowel. Stool studies including clostridium difficile PCR, bacterial culture, fecal leukocytes, Giardia antigen, occult blood, and ova and parasite testing all returned negative. Stool electrolyte results were as follows: stool sodium $104.30 \mathrm{mEq} / \mathrm{L}$, stool potassium $52 \mathrm{mEq} / \mathrm{L}$, and stool osmolality $316 \mathrm{mOsm}$. The calculated stool osmotic gap was $3 \mathrm{mOsm} / \mathrm{kg}$, suggesting a predominantly secretory-type diarrhea.

On admission, the olmesartan portion of her combination olmesartan medoxomil/hydrochlorothiazide (Benicar HCT) medication was interchanged to the pharmacy's formulary angiotensin II receptor blocker (ARB) candesartan, and the hydrochlorothiazide portion was held due to hypokalemia and dehydration. An inpatient gastroenterology consultation was requested due to her ongoing symptoms which led to severe electrolyte disturbances. On day 3 in hospital, the patient underwent a colonoscopy which was endoscopically normal besides diverticulosis in the sigmoid and distal descending colon. There was no evidence of bleeding, inflammation, polyps, or ulcerations. Biopsies throughout every segment of the colon in addition to the terminal ileum notably revealed inflammation within the lamina propria and superficial epithelium, associated with reactive and regenerative epithelial changes (Fig. 1), with results being available on day 5 in hospital. These findings were consistent with nonspecific lymphocytic colitis without any evidence of chronic inflammatory bowel disease.

On day 4 in hospital, computed tomography (CT) enterography was pursued to better evaluate the small bowel, though returned showing normal small and large bowel loops seen without evidence of mural thickening or abnormal enhancement. An esophagogastroduodenoscopy (EGD) was performed on day 5 in hospital, showing focal esophagitis in the upper third of the esophagus, mild nonspecific gastritis in the gastric antrum, and a normal-appearing duodenum. Biopsies had been obtained from the gastric antrum as well as the duodenum.

Given that her workup was largely unremarkable after her endoscopic procedures though with biopsy results still pending at that point, it was suspected that her olmesartan may be causing her symptomatology due to her profound and unremitting GI symptoms along with existing reports of olmesartan being a culprit drug in similar scenarios. Additionally, she had clinically improved over the days since she had been in the hospital while the drug was being held. The patient's home ARB olmesartan was switched to candesartan on admission, solely based on the automatic pharmacy interchange and the patient gradually improved in the interim, well enough to be discharged home on day 5. She was instructed not to resume her olmesartan medication and instead was provided with a prescription for candesartan. She was arranged for a followup appointment with the gastroenterologist for 1 month after discharge.

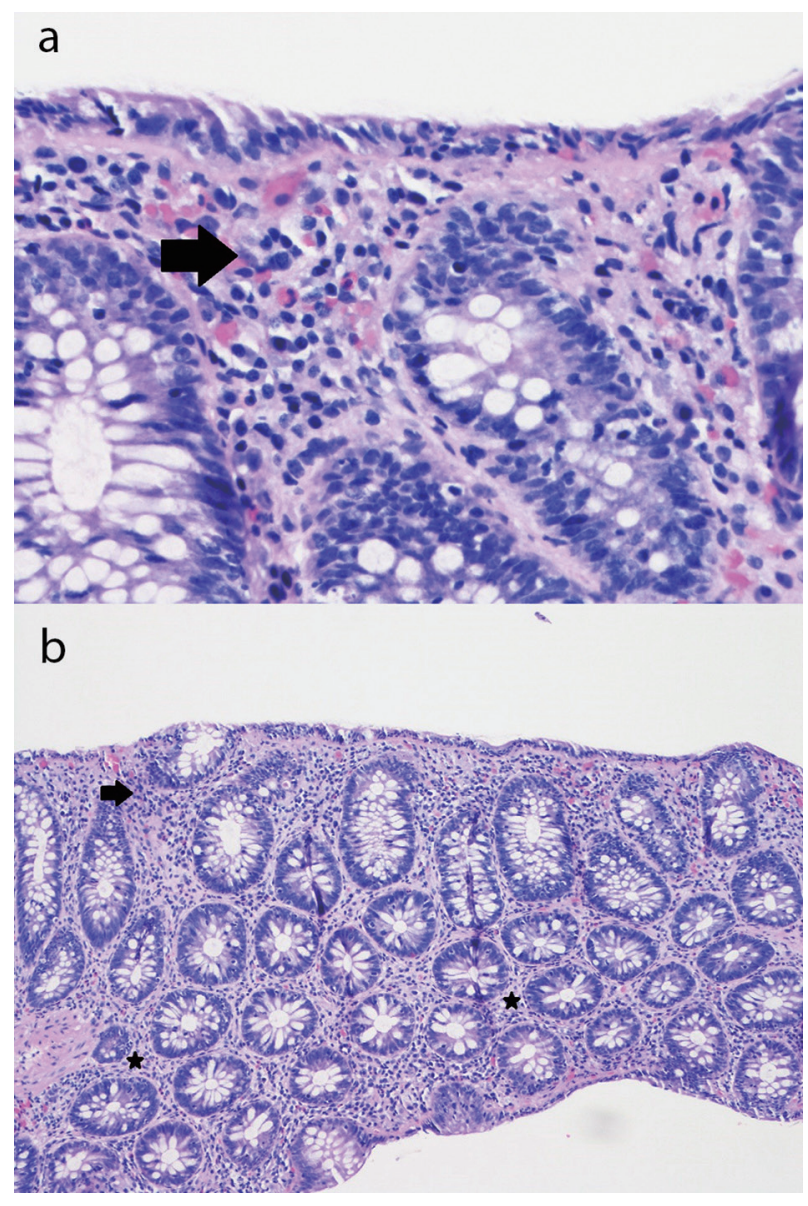

Figure 1. (a) Sigmoid colon mucosa (hematoxylin and eosin, $\times 400$ ) showing chronic inflammation in the superficial portion of the lamina propria and mild intraepithelial lymphocytosis with surface epithelial injury (lymphocytic colitis-like injury) with rare acute inflammatory cells in the lamina propria and surface epithelium (arrow). (b) Sigmoid colon mucosa (hematoxylin and eosin, $\times 100$ ) showing chronic inflammation in the superficial portion of the lamina propria and mild intraepithelial lymphocytosis with surface epithelial injury (lymphocytic colitis-like injury) with rare acute inflammatory cells in the lamina propria (stars) and surface epithelium (arrow).

Biopsy results from the EGD returned 2 days after the patient was discharged. It revealed chronic active gastritis in the gastric antrum with negative immunohistochemical stain for Helicobacter pylori. Biopsy obtained from the endoscopically normal duodenum showed chronic duodenitis with subtotal villous blunting, an increase in chronic inflammation of the lamina propria consisting of a mixed population of lymphocytes, plasma cells, and eosinophils as well as an intraepithelial lymphocytosis (Fig. 2). The notable findings from her duodenal biopsy results essentially confirmed our initial impressions of a drug-induced enteropathy, due to olmesartan.

After discharge and prior to her follow-up visit with the gastroenterologist, she had the candesartan medication switched to amlodipine and she was started on omeprazole for reflux symptoms. Tissue transglutaminase antibody testing returned negative. At her 1 month visit with the gastroenterologist, it was noted that she continued to clinically improve and 


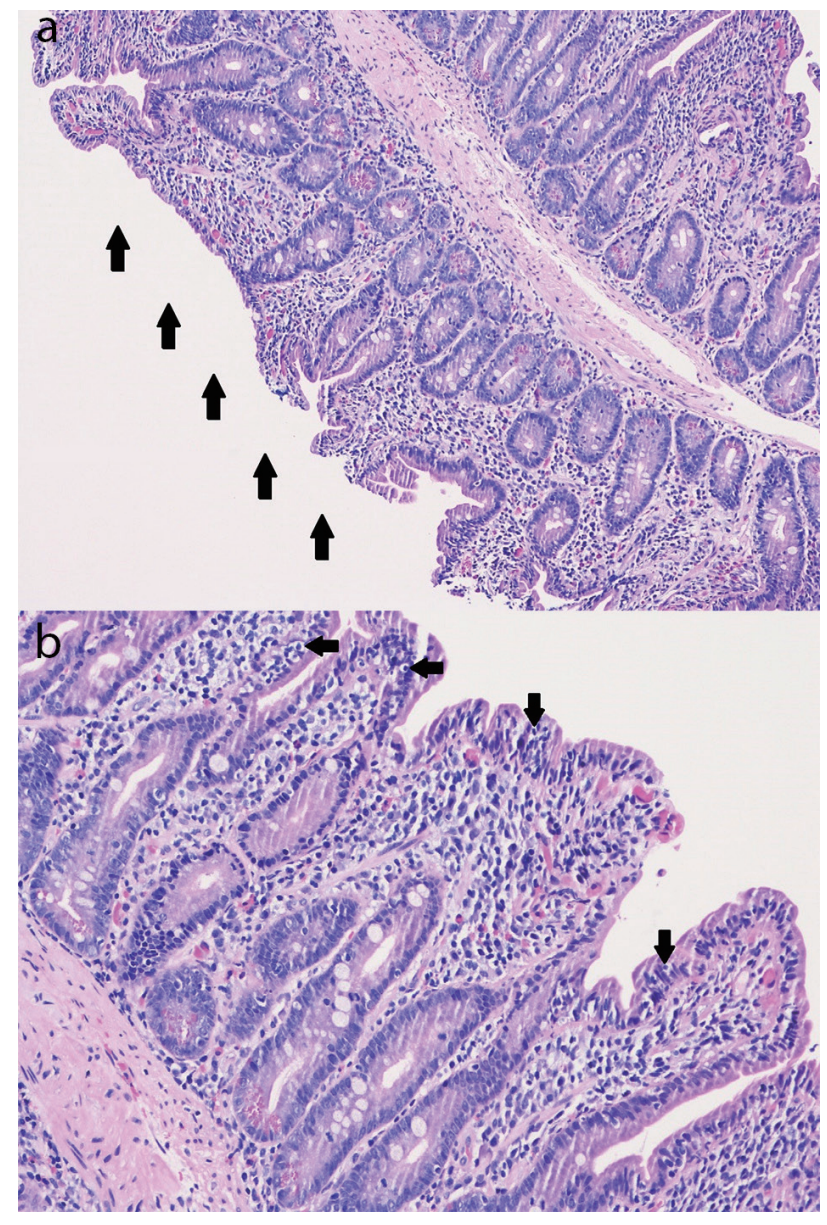

Figure 2. (a) Duodenal mucosa (hematoxylin and eosin, $\times 100$ ) with subtotal villous blunting (arrows) with chronic duodenitis with an increase in chronic inflammation of the lamina propria consisting of a mixed population of lymphocytes, plasma cells, and eosinophils as well as an increase in intraepithelial lymphocytes (intraepithelial lymphocytosis). (b) Duodenal mucosa (hematoxylin and eosin, $\times 200$ ) with subtotal villous blunting with chronic duodenitis with an increase in chronic inflammation of the lamina propria consisting of a mixed population of lymphocytes, plasma cells, and eosinophils as well as an increase in intraepithelial lymphocytes (intraepithelial lymphocytosis; arrows).

had no further weight loss. At that visit, it was made known that her granddaughter had just been recently diagnosed with celiac disease. The patient had another EGD 4 months after her hospitalization with duodenal biopsies revealing normal mucosa, histologically confirming resolution of the preexistent pathology.

\section{Discussion}

Olmesartan is one of several ARB drugs used for hypertension which was approved by the Food and Drug Administration (FDA) in 2002. In 2012, it was estimated that roughly 10.5 million prescriptions were dispensed for a single or combination olmesartan product in the USA [3]. A report of an association between olmesartan and drug-induced enteropathy was first published in August 2012 describing 22 patients evaluated from 2008 through 2010 at the Mayo Clinic [1]. Another study described villous atrophy and negative serologies for celiac disease in patients without definitive etiologies for their histological findings who were characterized as having unclassified sprue [2]. Some of these patients were later found to have villous atrophy associated with olmesartan use. As a result of this evidence, the FDA-approved label changes to olmesartancontaining products in July 2013 [4] to warn of an associated sprue-like enteropathy that may develop in patients.

A recent systematic review [5] analyzed all cases of ARBassociated enteropathy published between August 2012 and November 2018, which totaled 248. In almost all of the cases reviewed, olmesartan was the drug implicated, with the remaining 15 cases suspected to be secondary to other ARBs. There is no doubt that olmesartan is the ARB in which this adverse effect is most commonly observed, though based on the available cases, there is a suggestion that other ARBs may cause the same complication. Other ARBs in which sprue-like enteropathy has been associated include telmisartan, irbesartan, valsartan, losartan, and eprosartan [5]. The plausibility of a class effect being more substantive hinges on further studies. This is notable as the FDA hasn't come forward with any formal cautioning statements for other ARBs as it has with olmesartan, which may be the case in the future if and when more cases are recognized. In our case, the patient had initially been discharged home on candesartan, though her primary care physician then switched it to amlodipine, seemingly to avoid any possibility of a class effect.

In an overwhelming majority of cases of OAE the diagnosis may remain elusive, though fortunately, in our case, we arrived at the diagnosis fairly quickly. Making the diagnosis is particularly challenging and is usually delayed as seen in previously reported cases [1, 6-11]. Reasons for this are numerous and include the fact that patients present with a spectrum of nonspecific GI symptoms. These may include diarrhea, nausea, vomiting, abdominal pain, and bloating. Moreover, patients typically undergo several failed trials of various therapies prior to diagnosis, such as antibiotics, a change in diet, corticosteroids, and antimotility agents, among others. These various treatments and their possible adverse effects take their toll on patients who have to endure them without observed benefit. Furthermore, it has been described in previous cases that symptoms with accompanying pathology typically develop months to several years $[1,12]$ after initiation of the drug, which is another reason that the diagnosis may be unexpected and thus protracted. Usually, adverse drug reactions (ADRs) are assumed to occur relatively soon after their initiation, which would typically be more obvious to patients and their healthcare providers. Our patient had been on her combination olmesartan drug for at least 11 years before the onset of her symptoms which, to our knowledge, is the longest period of time between exposure to olmesartan and onset of symptoms.

Additionally, a crucial factor to consider that has occurred in previous cases $[1,6,7,10,11,13-15]$ but not particularly emphasized is how hospitalized patients frequently have their home olmesartan medication temporarily suspended prior to a formal diagnosis. Reasons for this include therapeutic interchange to an alternate ARB due to the institutional formulary, 
held for medical reasons such as hypotension or acute kidney injury, or held along with all other drugs for suspicion of a possible drug-induced phenomenon of unknown etiology. This interruption in turn acts as a distractor, allowing the patient to notice clinical improvement for reasons that may not be obvious to the treatment team only to have the drug subsequently resumed and further postpone the diagnosis.

Another circumstance which may delay diagnosis is when patients undergo endoscopy without biopsy, as has been previously described [16]. This may occur if endoscopic findings appear normal. Biopsies despite normal-appearing endoscopy is paramount in arriving at a formal diagnosis. EGD, and even colonoscopy, with corresponding random biopsies should be pursued during evaluation of this entity. Colitis noted histopathologically is a potential finding in a subset of patients with OAE, as was seen in our case, and described in others $[1,7,14]$ in the literature. Almost all patients that have been diagnosed with OAE have had EGD biopsies revealing total or partial villous atrophy and intraepithelial lymphocytosis as well as having negative serology testing for celiac disease [5].

Clinical improvement typically occurs within days to a week $[13,15,17]$ after olmesartan is suspended, as was seen with our case. Repeat EGD with biopsies 4 months after our patient's hospitalization confirmed histologic resolution of her enteropathy. Proving histologic recovery is not required in cases of OAE as long as the patient's symptoms clinically resolve off of the drug. If performed, biopsy typically shows histological improvement or remission in OAE relatively quickly, on the order of months $[1,17,18]$. Re-challenging patients with olmesartan is not recommended, though has occurred unknowingly in previous studies [7, 10, 11, 14] with resumption of symptoms exhibited. It is not unreasonable at this point in time to consider replacing a patient's olmesartan to an alternative ARB. This is an option if necessitating benefits attained from ARBs, though if this isn't a priority, then an agent from a different class may be the better option in order to avoid the possibility of a class effect altogether.

\section{Conclusions}

Olmesartan is a commonly prescribed antihypertensive drug, and over recent years it has been associated with severe sprue-like enteropathy which clinically presents with persistent nonspecific GI symptoms. In patients who present with unexplained chronic diarrhea and weight loss, clinicians must be cognizant of this rare but possible effect, as it is potentially life-threatening. Obtaining a comprehensive history including a focus on medications is of paramount importance when entertaining the possibility of a rare ADR. It is especially important to appreciate the many circumstances that may conceal, and in turn, delay the diagnosis. Diagnosis sooner rather than later may prevent avoidable hospitalizations, morbidity, and possibly even mortality. EGD with biopsy is recommended to identify the abnormal villous architecture observed in this condition. It is prudent to counsel patients and their families regarding this possible entity, especially as it may develop after months to several years after the drug has been initiated.

\section{Acknowledgments}

The author wishes to thank Dr. Philip McDowell of Department of Pathology at Greater Baltimore Medical Center, for his expert descriptions and preparation of the images within the manuscript.

\section{Financial Disclosure}

None to declare.

\section{Conflict of Interest}

None to declare.

\section{Informed Consent}

The informed consent was obtained by the treating physicians.

\section{Author Contributions}

DRB contributed to the conception, literature review, drafting, and revising the manuscript.

\section{Data Availability}

The author declares that data supporting the findings of this study are available within the article.

\section{References}

1. Rubio-Tapia A, Herman ML, Ludvigsson JF, Kelly DG, Mangan TF, Wu TT, Murray JA. Severe spruelike enteropathy associated with olmesartan. Mayo Clin Proc. 2012;87(8):732-738.

2. DeGaetani M, Tennyson CA, Lebwohl B, Lewis SK, Abu Daya H, Arguelles-Grande C, Bhagat G, et al. Villous atrophy and negative celiac serology: a diagnostic and therapeutic dilemma. Am J Gastroenterol. 2013;108(5):647653.

3. Intercontinental Medical Statistics. IMS, Vector One: National (VONA) and Total Patient Tracker (TPT) database. 2012. Extracted June 2013.

4. United States Food and Drug Administration. FDA Drug Safety Communication: FDA approves label changes to include intestinal problems (sprue-like enteropathy) linked to blood pressure medicine olmesartan medoxomil. July 3, 2013. http:/www.fda.gov/Drugs/DrugSafety/ucm359477.htm. Accessed May 26, 2020.

5. Kamal A, Fain C, Park A, Wang P, Gonzalez-Velez E, Leffler DA, Hutfless SM. Angiotensin II receptor blockers 
and gastrointestinal adverse events of resembling spruelike enteropathy: a systematic review. Gastroenterol Rep (Oxf). 2019;7(3):162-167.

6. Dreifuss SE, Tomizawa Y, Farber NJ, Davison JM, Sohnen AE. Spruelike enteropathy associated with olmesartan: an unusual case of severe diarrhea. Case Rep Gastrointest Med. 2013;2013:618071.

7. Theophile H, David XR, Miremont-Salame G, Haramburu F. Five cases of sprue-like enteropathy in patients treated by olmesartan. Dig Liver Dis. 2014;46(5):465469.

8. Abdelghany M, Gonzalez L, 3rd, Slater J, Begley C. Olmesartan associated sprue-like enteropathy and colon perforation. Case Rep Gastrointest Med. 2014;2014:494098.

9. Naik DK, Martelli MG, Gonzalo DH, Sharma AK, Pannu D. An atypical case of chronic diarrhoea: olmesartan-induced sprue-like enteropathy. BMJ Case Rep. 2015;2015.

10. Hammoudi N, Dior M, Giraud V, Coffin B. Olmesartaninduced enteropathy associated with cutaneous lesions. Clin Case Rep. 2016;4(4):379-382.

11. Uehara T, Ikusaka M, Ohira Y, Noda K, Suzuki S, Shikino $\mathrm{K}$, Kondo T, et al. Olmesartan-induced enteropathy manifesting as Wernicke-Korsakoff syndrome. Intern Med. 2016;55(24):3675-3678.

12. Aderinto A, Colman R, Quigley E. Not all diarrhea and villous atrophy is celiac disease: a case report on olmesartan-induced enteropathy. Am J Gastroenterol. 2014;109:S333-334.

13. Stanich PP, Yearsley M, Meyer MM. Olmesartan-associated sprue-like enteropathy. J Clin Gastroenterol. 2013;47(10):894-895.

14. Marthey L, Cadiot G, Seksik P, Pouderoux P, Lacroute J, Skinazi F, Mesnard B, et al. Olmesartan-associated enteropathy: results of a national survey. Aliment Pharmacol Ther. 2014;40(9):1103-1109.

15. Shahzad MA, Harding D, Ruszkiewicz A, Tran E, England G, Philpott H. Gastrointestinal: Olmesartan-induced enteropathy. J Gastroenterol Hepatol. 2018;33(10):1691.

16. Solano-Iturri G, Garcia-Jimenez N, Solano-Iturri JD, Blanco-Sampascual S. Olmesartan-associated sprue-like enteropathy: An emerging cause of druginduced chronic diarrhea. Rev Gastroenterol Mex. 2018;83(1):71-72.

17. Choi EY, McKenna BJ. Olmesartan-associated enteropathy: a review of clinical and histologic findings. Arch Pathol Lab Med. 2015;139(10):1242-1247.

18. Nielsen JA, Steephen A, Lewin M. Angiotensin-II inhibitor (olmesartan)-induced collagenous sprue with resolution following discontinuation of drug. World J Gastroenterol. 2013;19(40):6928-6930. 